\title{
Beobachtung zweier Fälle einer noch wenig beschriebenen chronischen katarrhalischen Wurmfortsatzentzündung.
}

\author{
Von
}

G. 0. E. Lignac.

ehem. Lehrer der Allgemeinen Pathologie, Pathologischen Anatomie und Gerichtlichen Medizin an der medizinischen Schule zu Batavia, jetzt Pros. am Path. Inst. Leiden.

\section{Mit' 4 Textabbildungen.}

Die chronische katarrhalische Wurmfortsatzentzündung ist eine Erkrankung, welcher der Chirurg sowie der Patholog-Anatom mehrmals begegnet. Jedoch kann unter gewissen Umständen (die ich nachher erörtern werde) die anatomische Abweichung eine so eigenartige sein,

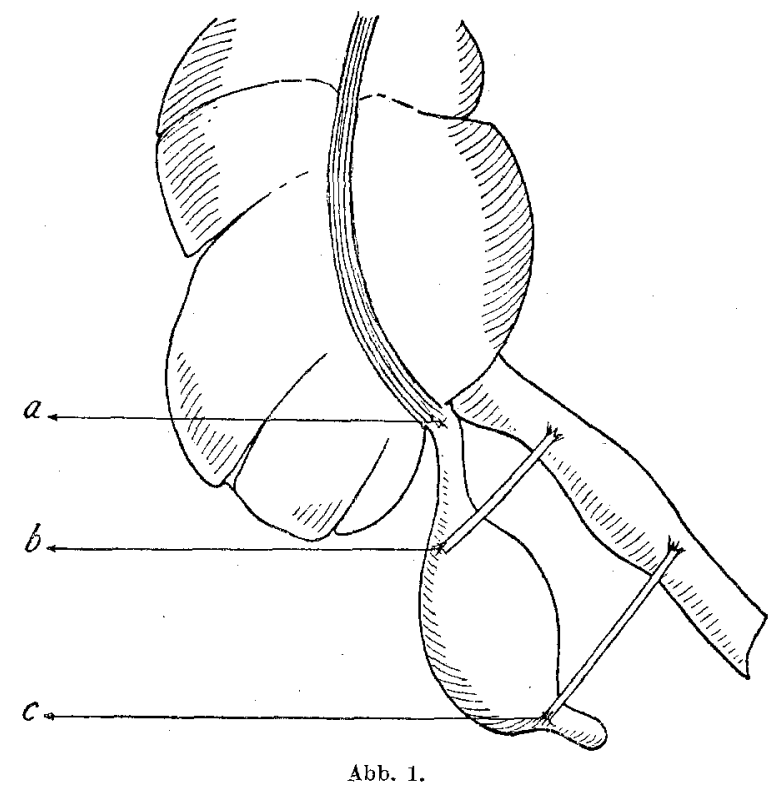

daß man fleißig in der betreffenden Literatur herumsuchen muß, ob vielleicht schon andere Beobachter dieselbe gefunden haben.

Bevor ich die Mitteilungen anderer in bezug auf diesen Gegenstand wiedergebe, will ich den anatomischen Befund der zwei bei der Obduktion von Malaien beobachteten Fälle einer chronischen katarrhalischen Wurmfortsatzentzündung berichten. 
Fall 1. Zur Obduktion (12 Stunden nach dem Tode) kam ein Malaie, mutmaßlich 75 Jahre alt, 1.72 m lang; das Fettgewebe war sehr schwach entwickelt, die Haut glatt und glänzend; es fand sich eine bogenförmige Kyphose. Beim Öffnen der Bauchhöhle fand ich nach erfolgter Untersuchung, daß die Form des Wurmfortsatzes sehr von der Norm abwich. Abb. 1 stellt den Wurmfortsatz mit Umgebung schematisch dar. Man sieht den Wurmfortsatz halbmondförmig gekrümmt mit der konkaven Seite nach links gerichtet; von der Oberfläche aus ziehen sich zwei fadenförmige Adhäsionen nach dem. Ende des Ileums (Verwechselung mit den Plicae ileo-caecales in casu ausgeschlossen). Wie man sieht, zeigt der Processus vermiformis in seinem mittelsten Teile eine spindelförmige Er-

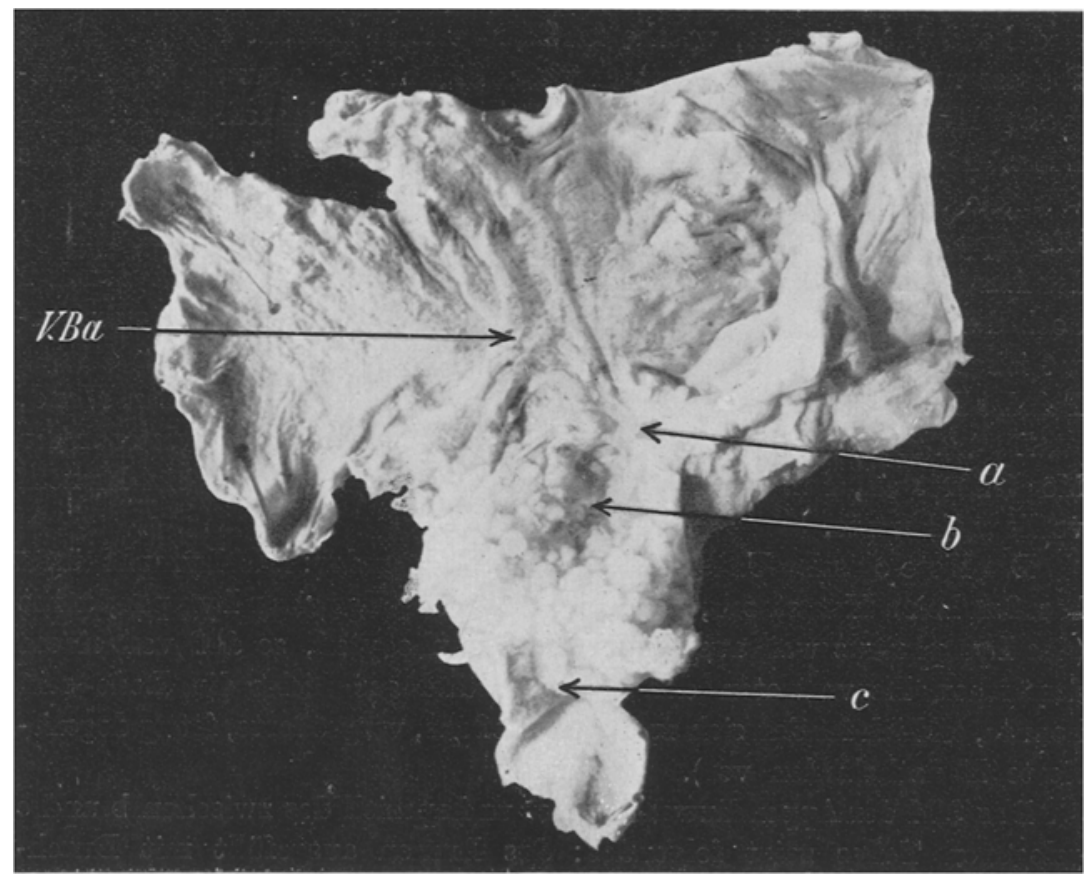

Abb. 2.

weiterung zwischen $b$ und $c$. Hier konnte man zwei leistenförmige Verdickungen fühlen, während zwischen $b$ und $c$ die Wand des Processus bläulich durchscheinend aussah. Die regionären Lymphdrüsen waren angeschwollen, fühlten sich mäßig fest an und zeigten auf dem Durchschnitte eine graurote Farbe. (Diese wurden auch für die mikroskopische Durchmusterung aufbewahrt.) Weiter wurden keine Abweichungen, weder am visceralen noch am parietalen Peritoneum gefunden; nur eine Spur von hellgelber Flüssigkeit war in der Bauchhöhle vorhanden.

Was das weitere Resultat der Obduktion anbetrifft, kann ich mich kurz fassen. Herz, Leber, Nieren, graue Hirnsubstanz zeigten starke braune Atrophie, die Lungen waren senil emphysematös. Herz und Gefäße enthielten speckbäutige Gerinnsel.

Der Mann war an Marasmus senilis gestorben.

Abb. 2 zeigt uns das von hinten geöffnete Coecum, Ileum und Processus. Das Präparat, ist photographiert worden, während die Sonnen- 
strahlen quer durch die schleimige Masse innerhalb des Processus hindurch geworfen wurden. Die Valvula ileocoecalis ist mit $\mathrm{V}$. Ba, die vollständig obliterierte Mündungsstelle des Wurmfortsatzes mit a und die in das Lumen vorragenden Leisten sind mit $b$ und $c$ bezeichnet. Zwischen $a$ und $b$ befindet sich der erste dritte Teil des Processus, wovon das Lumen erweitert und die Wand verdickt ist. Nur durch eine sehr kleine Öffnung ,kommuniziert das Lumen mit dem des mittlern dritten Teiles. Hier sehen wir, daß das stark spindelförmig erweiterte Lumen ausgefüllt ist mit einer grauen, durchscheinenden, äußerst adund kohärenten Masse, welche in $3 \mathrm{~mm}$ dicker Schicht die Wand des Processus bekleidet und womit an vielen Stellen kleine runde, hagelkorngroße Kügelchen mittels dünner Stielchen zusammenhängen. Der Zusammenhang ist ein nicht allzufester, so daß bei der Öffnung des Wurmfortsatzes mehrere Schleimkügelehen aus dem Processus rollten. Die Appendixwand ist hier, obgleich verdickt, relativ dünner infolge der Ausdehnung durch die Anhäufung des Schleimes, der weder durch $b$ noch durch $\mathrm{c}$ genügend, ja selbst gar nicht aus dem Wurmfortsatz ausgetrieben werden kann. Schließlich sieht man unter c den letzten dritten Teil des Processus vermiformis ebenso erweitert und mit verdickter Wand.

$\mathrm{Da}$ ich niemals diese Abweichung gesehen hatte, nahm ich noch die Möglichkeit an, daß ein schleimformendes Careinom oder ein Myxom vorliegen konnte.

Die histologische Untersuchung lehrte folgendes: Die Lymphdrüsen besaßen keine Metastasen, waren vergrößert infolge einer Lymphadenitis (der Lymphsinus war ausgefüllt mit Lymphocyten, so daß von einem deutlichen Hervortreten der Lymphfollikel gar nicht die Rede sein konnte, endlich fanden sich auch abgestoßene, vergrößerte, fettig degenerierte Endothelzellen vor).

Das Bild der Appendixwand selbst und speziell des zwischen b und $c$ gelegenen Teiles zeigt folgendes: das Lumen ausgefüllt mit Durchschnitten der Kügelchen, aus einem Geflecht feiner Fäden bestehend, welche sich nach der Peripherie zu konzentrisch aneinanderreihen und zusammengedrängt sind. Zwischen den Fäden sieht man ab und zu Lymphocyten oder polymorphkernige Leukocyten (besonders eosinophile) oder durch Eosine hellrot gefärbte Zellen, wovon die Kerne nur als Schatten gesehen werden. Die Fäden färben sich mit Thionin rotviolett (Schleimreaktion). Wie gesagt hängen die Kügelchen mittels feiner Fäden mit einer Schleimschicht zusammen, welche im Gegensatz zu den Kügelchen alle möglichen Stadien der jungen Fibroblasten bis zum feinen fibrillären Bindegewebe zeigt. Diese Schicht reiht sich der Submucosa an, da nirgends Epithel oder Krypten gefunden werden, außer vielleicht den nekrotischen Utberbleibseln derselben. In der Submucosa sieht man noch ab und zu Lymphfollikel, dazwischen zerstreut 
Lymphocyten, Plasmazellen, Leukocyten und Fibroblasten; darauf folgend sehen wir die Ring- und Längsmuskulatur aus glatten Muskelzellen bestehend, welche zu dünnen Fäden geworden sind, worin hie und da kaum ein Kern zu sehen ist; auch hier wieder fein fibrilläres Bindegewebe und einige zerstreute Lymphocyten (differenziert mit van Gieson). Die glatten Muskelzellen zeigen Dehnungsatrophie. Vermehrung des Bindegewebes mit perivasculären Lymphocytenanhäufungen unter dem Peritoneum ist auch zu sehen.

Wir haben hier also vor uns eine chronische katarrhale Entzündungdes Wurmfortsatzes, wobei infolge teilweisen Verschlusses des mittleren Teiles Anhäufung des Schleimes besonders da stattgefunden hat, welchem Umstand die so starke Dehnung desselben zuzuschreiben ist. Die Wand ist nirgends eingerissen; die Entzündung hat den ganzen Wurmfortsatz ergriffen und die totale Obliteration der Valvula von Gerlach herbeigeführt. Ich will noch hervorheben, daß das schleimige Sekret hier in eigenartiger Form erscheint. Von Hansemann (nachher wird noch weiter darüber gesprochen werden) nennt diese Gebilde My xoglobulosen. Inwiefern noch schleimige Degeneration des Bindegewebes hinzukommt, lasse ich dahingestellt.

Innerhalb eines halben Jahres begegnete ich einem zweiten Falle, welchen ich hier kurz erwähnen will.

Zur Obduktion (24.Stunden nach dem Tode) gelangte ein mir unbekannter Nalaie, mutmaßlich 30 Jahre alt, 1,60 m lang und kräftig gebaut. Was die anatomischen Abweichungen anbetrifft, so nenne ich die Zeichen der fibrinösen Pneumonie in der rechten Lunge: graue Hepatisation der zentralen und rote Hepatisation der caudalen Teile mit sero-fibrinöser Pleuritis; Pigmentmilz, starke Vermehrung des Bindegewebes und da. Verwesung eingetreten war, konnte ich den wirklichen Zustand der Pulpa nicht beurteilen (das Gewicht $=620 \mathrm{~g}$ ); die Leber zeigte auf dem Durchschnitte, obgleich nicht zusammengeschrumpft (Gewicht $=1480 \mathrm{~g}$ ) schöne Pseudo-acini (die mikroskopische Durchmusterung der Präparate lehrte, daß eine atrophische Lebercirrhose vorlag).

An dem visceralen und parietalen Peritoneum wurden keine Abweichungen gefunden, in der Bauchhöhle befand sich nur ein sehr geringes Quantum einer hellen, braungefärbten Flüssigkeit.

Gehen wir jetzt zur Beschreibung des Wurmfortsatzes mit Umgebung über:

Der proximale Teil des Processus ist mit dem Ende des Mleums mittels einer starken Bindegewebsmembran verbunden (in Abb. 3 nicht gezeichnet). Der distale Teil des Processus, dreiviertel des Ganzen, ist ballonförmig gespannt und der Inhalt desselben kommuniziert mit der schussergroßen Höhle einer Cyste, welche sich zwischen den beiden Blättern des Mezenteriolums befindet und an deren Oberfläche sich eine leichte Einschnürung zeigt.

Der Wurmfortsatz mit seiner Umgebung wird von der freien Seite dem Ansatze des Mesenteriolums gegenüber aufgeschnitten (Abb. 4). Beim Aufschneiden rollen kleine kaviargroße noch miteinander zusammenhängende Kügelcher ( $\mathrm{I}$ in Abb. 4) aus dem Processus. Man sieht auch die total obliterierte Mündung des Wurmfortsatzes mit V. G. angegeben. Aus dem ballonförmig angeschwollenen Teile des Processus quillt eine schleimige Masse heraus, worin man hier und da noch kleine Kügelchen sehen kann (auf der Photographie sieht 
die Masse etwas verwischt aus). Das schleimige Sekret hängt nieht nur miţ der Wand des Wurmfortsatzes, sondern auch mit der der Cyste zusammen. Man fühlt und sieht jetzt auch, daß ein dicker WaIl die Kommunikationsstelle des Processus und Cyste ringförmig umschließt. Die ganze Wand des Wurmfortsatzes ist verdickt und auch hier wieder sind die regionären Lymphdrüsen angeschwollen.

Die histologische Untersuchung kann kurz gefaßt werden, da auch hier wieder Zeichen einer chronischen katarrhalischen Entzündung vorhanden waren. Die Wand der intramesenterialen Höhle $-d$. h. der sog. Cyste - besa 3 eine Schleimschicht, eine Bindegewebsmembran,

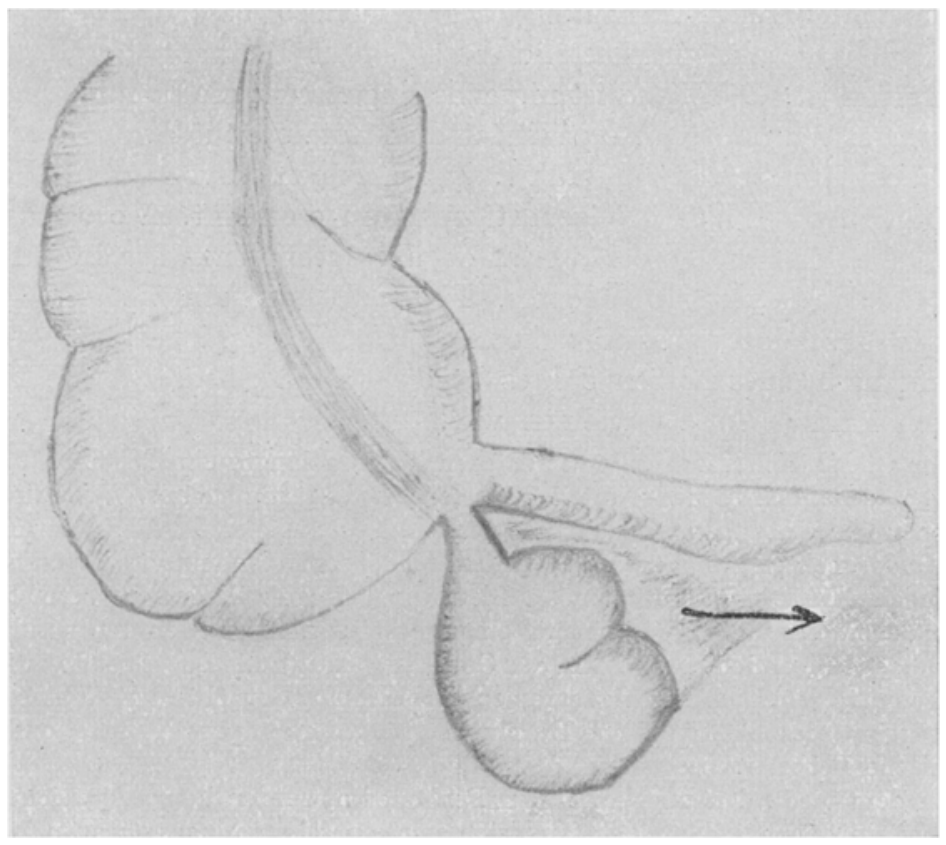

Abb. 3.

einzelne Fettzellen, subperitoneales Bindegewebe mit Nerven, Gefäßen und endlich die peritoneale Endothelschicht.

Wie die Höhle im Mesenteriolum entstanden ist, scheint mir in folgender Weise zu erklären möglich. Durch starke Ausdehnung des distalen Teiles des Processus infolge Schleimanhäufung ist Atrophie der Wand eingetreten (Spuren davon sind deutlich zu sehen) und ist diese auf einmal an der Seite des Ansatzes des Mesenteriolums (vielleicht durch plötzliche oder allmähliche Druckerhöhung) an der schwächsten Stelle - hat hier schleimige Degeneration des Bindegewebes vielleicht mitgewirkt? - eingerissen. Der Inhalt, zwischen den Lamellen des Peri- 
toneums des Processus hindurchgepreßt, hat hier eine Höhle mitten im lockeren Bindegewebe gebildet.

Auch in diesem Falle nirgends maligne Neubildung, die regionären Lymphdrüsen entzündet.

Hervorheben will ich, daß ich weder im ersten Falle noch im zweiten an der Appendixwand, wo das Epithel vorhanden war, Cysten der Krypten habe finden können. Die Fpithelzellen waren da fast nicht gefärbt oder gar nicht anwesend, die Krypten oft mangelhaft ausgebildet.

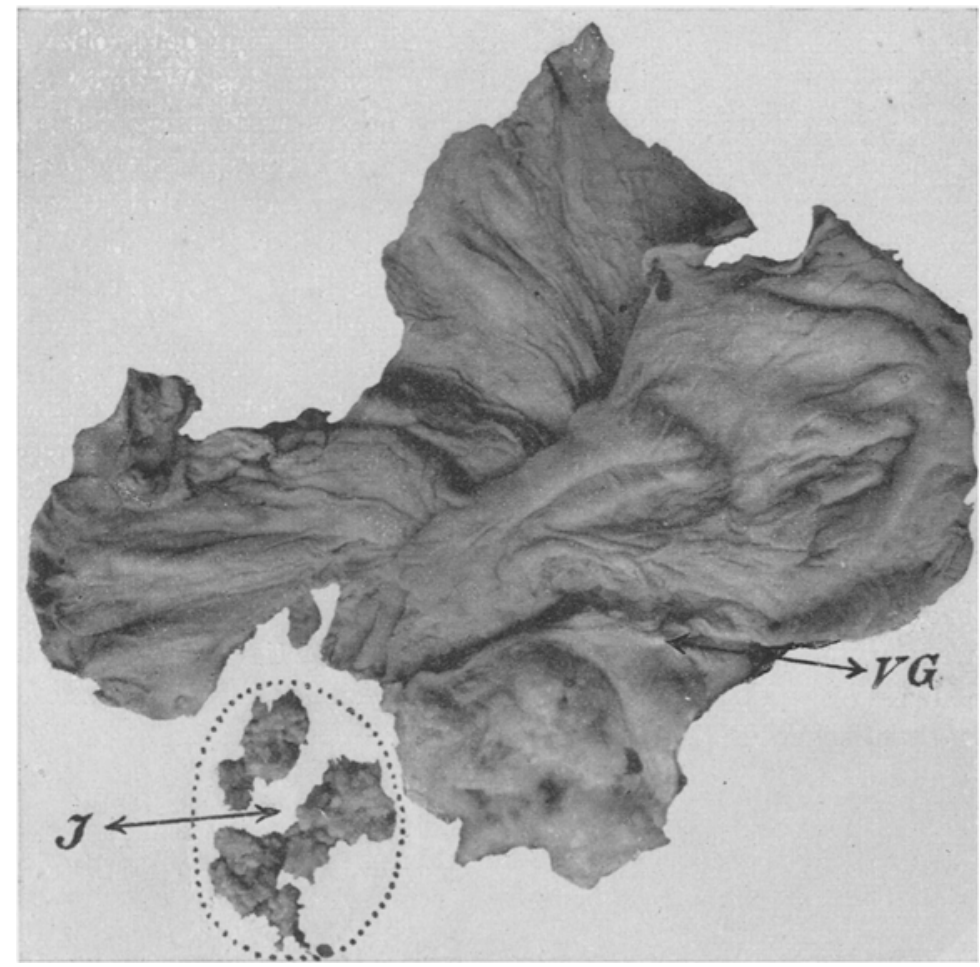

Abb. 4.

Wenden wir uns jetzt zur Literatur, so haben wir auch Gelegenheit die Genese der Schleimkügelchen za besprechen. Roth ${ }^{\mathbf{1}}$ ) teilt uns mit, daß von Hansemann auf der Pathologentagung (1915) zwei verschlossene Wurmfortsätze, welche mit lauter kleinen gallertigen Kugeln von Kaviargröße vollständig gefüllt waren, beschrieben hat. Ein Teil der Kugeln lag nach Perforation der Appendix in einer durch Adhäsion gebildeten Tasche. Die kleinen Gebilde werden in erweiterten Schleimdrüsen gebildet und dann vergrößert. Von Hansemann bezeichnet diese Erscheinung als Myxoglobulose und gibt an, daß sein Befund 
in der Literatur drei Vorläufer hat, nämlich die Fälle Cagnettos, Chavannaz' und Sabrazès'. Leider habe ich nur Roths Referat und Cagnettos Befund ${ }^{2}$ ) habhaft werden können und ich will darum Cagnettos Mitteilung hier ausführlich durchnehmen, weil diese zur Erklärung meines Befundes notwendig ist.

Der veränderte Wurmfortsatz gehörte einer 70jährigen Frau, deren Leiche nicht nur die durch das Alter bedingten Veränderungen, sondern auch eine kürzlich überstandene rechtsseitige serofibrinöse Pleuritis aufwies.

Der Processus ist stark ausgedehnt und an Umfang der großen Zehe eines Erwachsenen gleich. Die Mündungsstelle des Wurmfortsatzes ist durch eine bläulichgraue vertieft liegende, sternförmige Narbe von der Größe einer Linse verschlossen, während die Eingangsstelle des Processus an der Basis des Blinddarms fest verwachsen ist und dort die Wand der letzteren einwärts gedrängt hat. Beim Öffnen des Wurmfortsatzes zeigt sich der Inhalt, welcher aus lauter traubenförmig zusammengefügten, kleinen, bläschenartigen gelatinösen Kügelchen, von einer fadenziehenden Substanz zusammengehalten, besteht. Die Schleimhaut des Wurmfortsatzes ist über ihre ganze Oberfläche mit einer Schicht zähen, klebrigen und dicken Schleimes bedeckt, nach dessen Entfernung zahlreiche rötliche Flecke infolge der Hyperämie zutage treten, die mit graugefärbten Stellen abwechseln. Es gibt Stellen an der Appendixwand, wo die letztere so dünn wie Pergament und beutelartig nach außen ausgedehnt ist.

Cagnetto will die Natur dieser bläschenartigen Bildungen feststellen und fragt, ob man es hier mit parasitären Cysten oder vielmehr mit pseudocystischen Bildungen zu tun habe.

Die grobanatomische sowie histologische Untersuchung schließt parasitäre Cysten aus, und ich kann mitteilen, daB der Befund Cagnettos sich größtenteils deckt mit dem meinigen; nur eine Tatsache will ich besonders hervorheben:

Wo Cagnetto die Schleimhaut und besonders die Stellen, wo es noch sichtbare Drüsen gibt, beschreibt (S. 202), sagt er :

„Da und dort beobachtet man auch ein kleines rundliches cystenartiges Gebilde, das durch eine dünne bindegewebige Schicht vom Oberflächenepithel der Schleimhaut getrennt ist und höchstwahrscheinlich durch die dauernde Obliteration der Eingangsstelle hervorgerufen wird."

Diese Tatsache habe ich in meinen beiden Fällen nicht feststellen können und doch ist diese so notwendig zur Erklärung des eigentümlichen Gebildes. Ich glaube denn auch, daß diese zwei Fälle weiter vorgeschritten sind als der Cagnettos. Daß Cagnetto die cystenartige Umwandlung der Schleimdrüsen durch Ansammlung des Sekretes nur 
seltèn gesehen hat, zeigt sich, wo der Autor den Mechanismus (S. 208) bespricht:

- „Bei der einen handelt es sich um eine Stenosis der Mündungen der Schleimdrüsen und die darauf folgende Ansammlung eines Teiles des Sekretes. Der Genauigkeit halber will ich erwähnen, daß hinreichend klare Merkmale dieses Vorganges nur in wenigen meiner Präparate sichtbar sind."

Cagnetto gibt weiter an, wie er sich die Genese des ganzen Gebildes denkt, und ich will diese hier kurz wiedergeben:

1. Sollen die kleinsten kugelförmigen Bildungen durch die Anhäufung des Schleimes infolge Stenosis der Mündungen der Schleimdrüsen entstehen, bis endlich die ganz kleinen Klümpchen ausgeschieden werden oder auch verschmelzen können (3-4 z. B.).

2. Sollen die großen Konglomerate entstehen wie die sog. Reiskörperchen in der entzündeten Sehnenscheide [Schuchard $\left.\mathbf{t}^{3}\right), \mathrm{König}^{4}$ ), ${ }^{5}$ ) und $\left.{ }^{6}\right)$ ]. Nämlich sollen losgelöste Schleimfetzen infolge der schiebenden und rotierenden Bewegung, durch die Peristaltik des Appendix bedingt, zusammengerollt werden und so die kugeligen und geschichteten Formen entstehen.

Folgende Bemerkung will ich diesen Ausführungen noch hinzufügen, nämlich, daß eines oder mehrere der ausgeschiedenen Schleimklümpchen zusammen Kerne bilden können und daß durch Apposition von Schleimfetzchen diese ursprünglich winzigen Klümpchen zur Kaviar-, ja selbst Hagelkorngröße anwachsen können unter der Bedingung jedoch, daß durch die peristaltische Bewegung des Appendix die Klümpchen immer wieder zur Kugelform geknetet werden. Ob im jetzigen Zustande bei derartiger Atrophie noch eine Peristaltik besteht, glaube ich kaum. Wir haben hier weit vorgeschrittene Fälle, wenn man will Endstadien (die Entzündung, obschon chronisch, ist doch noch vorhanden), weil die soeben genannte Genese der Gebilde eine ist, welche sich während der katarrhalischen, später chronisch gewordenen Entzündung vollzogen hat. Cagnetto vermutet, daß eine katarrhalische Entzündung des Coecum auf den Wurmfortsatz übergegangen ist. Dies ist ganz wohl möglich, da wir wissen, daß die oberflächliche, exsudative, diffuse Entzündung der Schleimhäute (Katarrh) besonders gerne sich der Oberfläche nach ausbreitet. Die Zeichen einer katarrhalischen Wurmfortsatzentzündung sind noch zugegen, nur ist die Entzündung im Wurmfortsatz chronisch geworden. (Ich erinnere hier an die Colitis cystica, wobei sich derselbe Vorgang abspielt wie an den besprochenen Wurmfortsätzen.) Welche Rolle dürfte hier noch eine etwaige hinzugekommene schleimige Degeneration des Bindegewebes spielen?

Weiter führt Cagnetto an, daß vielmehr eine Typhlitis als eine primäre Appendicitis Veranlassung zum Verwachsen der Gerlachschen 


\section{G. O.E. Lignac:Beobachtung zweier Fälle einer noch wenig beschriebenen usw.}

Falte gegeben hat. Sind die Adhäsionen in meinen beiden Fällen vielleicht die Reste einer Perityphlitis oder die Produkte der chronisohen Appendicitis?

Der Verschluß an der Mündungsstelle des Wurmfortsatzes ist eine wichtige Tatsache in der Genese dieser Gebilde und man findet die auch angegeben in den beiden Fällen Hansemanns, im Falle Cagnettos und in den beiden meinigen. Der Verschluß macht die Fortschaffung des Sekretes aus dem Appendix unmöglich und den Wurmfortsatz erst recht zu einem toten Raume, wodurch die Entzündung auch hier lokalisiert bleibt.

In der Genese spielt die nicht hinreichende flüssige Zufuhr auch eine große Rolle, so sagt wenigstens Cag netto und weist dabei auf die Entstehung der Kotsteine des Wurmfortsatzes und der Konkremente im übrigen Eingeweide hin.

Inwiefern diese Wurmfortsätze zu Beschwerden zeitlebens Veranlassung gegeben haben, kann ich nicht erörtern. Die beiden Malaien wurden kurze Zeit vor dem Tode ins Spital aufgenommen.

Zum Schlusse will ich noch erwähnen, daß Wurmfortsätze, wie sie hier beschrieben sind, nach dem Bersten mit auffolgender Entleerung des Inhaltes in die freie Bauchhöhle zum ,Pseudomyxoma e Processu vermiformi" Anlaß geben dürften. (Roth 1.c.).

\section{Literaturverzeichnis.}

1) Roth, Beiträge z. pathol. Anat. u. allg. Path. (Ziegler) 61, 58. Heft 1, 1915. $\left.-{ }^{2}\right)$ Cag net to, Virchows Archiv 198, 193. Heft 2, 1909. - $\left.{ }^{3}\right)$ Sch uchardt, Virchows Archiv 114, 186. Heft 1, 1888. - $\left.{ }^{4}\right) \mathrm{König,} \mathrm{Die} \mathrm{Tuberkulose} \mathrm{der} \mathrm{Knochen}$ und Gelenke. Berlin 1884, - 5) König, Zentralbl. f. Chir. 1886, S. 424-430. Nr. 25. - ${ }^{6}$ ) König, Lehrbuch der speziellen Chirurgie 3, 148. 1889.

Den ersten Fall habe ich in ,Het Geneeskundig Tijdschrift voor Nederlandsch-Indie", Deel LVIII, 1918, S. 897, veröffentlicht. 